\title{
In Vivo Recording of Single-Unit Activity during Singing in Zebra Finches
}

\author{
Tatsuo S. Okubo, Emily L. Mackevicius, and Michale S. Fee ${ }^{1}$ \\ Department of Brain and Cognitive Sciences, McGovern Institute for Brain Research, Massachusetts Institute \\ of Technology, Cambridge, Massachusetts 02139
}

The zebra finch is an important model for investigating the neural mechanisms that underlie vocal production and learning. Previous anatomical and gene expression studies have identified an interconnected set of brain areas in this organism that are important for singing. To advance our understanding of how these various brain areas act together to learn and produce a highly stereotyped song, it is necessary to record the activity of individual neurons during singing. Here, we present a protocol for recording single-unit activity in freely moving zebra finches during singing using a miniature, motorized microdrive. It includes procedures for both the microdrive implant surgery and the electrophysiological recordings. There are several advantages of this technique: (1) high-impedance electrodes can be used in the microdrive to obtain well-isolated single units; (2) a motorized microdrive is used to remotely control the electrode position, allowing neurons to be isolated without handling the bird, and (3) a lateral positioner is used to move electrodes into fresh tissue before each penetration, allowing recordings from well-isolated neurons over the course of several weeks. We also describe the application of the antidromic stimulation and the spike collision test to identify neurons based on the axonal projection patterns.

It is essential that you consult the appropriate Material Safety Data Sheets and your institution's Environmental Health and Safety Office for proper handling of equipment and hazardous material used in this protocol.

\section{Reagents}

Analgesic (e.g., buprenorphine, $0.05 \mathrm{mg} / \mathrm{kg}, 0.75 \%$ in phosphate-buffered saline [PBS])

Betadine solution (10\% in distilled water)

Dental acrylic (e.g., Flow-It Accelerated Light Cure from Pentron Clinical)

General anesthetic (e.g., isofluorane)

Hypodermic needle (30-gauge; e.g., 305106 from BD)

Liquid soldering flux for stainless steel (e.g., Stay-Clean from Harris Products Group)

Local anesthetic (e.g., bupivacaine, $0.2 \mathrm{mg} / \mathrm{kg}, 0.05 \mathrm{mg} / \mathrm{mL}$ in PBS)

Silicon elastomer (e.g., Kwik-Cast from World Precision Instruments)

Tissue adhesive (e.g., Vetbond from 3M)

\footnotetext{
${ }^{1}$ Correspondence: fee@mit.edu 


\section{T.S. Okubo et al.}

\section{Equipment}

See Figure 1 for a schematic overview of the equipment required for the electrophysiological recording setup and the microdrive implant.

Amplifier for neural signals with a gain of 100 (e.g., TL084 from Texas Instruments)

Amplifier used during surgical setup (e.g., Model 1800 from A-M Systems)

Audio monitor (e.g., AM10 from Grass Technologies)

Birdcage $(\sim 23 \mathrm{~cm}$ in diameter and $\sim 20 \mathrm{~cm}$ in height)

Cable

The cable should have a male connector with wires, a female connector (e.g., A7876-001 and A7877-001, respectively, from Omnetics Connector), and surface-mount field-effect transistors (FETS; e.g., 2SK3796-3-TL-E, from ON Semiconductor) in a source-follower configuration on the microdrive side of the cable (see Fig. 2C, D).

Collision test circuit

This is an electrical circuit that triggers the pulse generator on detecting a threshold-crossing event owing to an occurrence of a spike. It consists of an operational amplifier (e.g., TL084 from Texas Instruments), an inverter chip (e.g., SN74LS04N from Texas Instruments), a timer chip (e.g., LM555 from Fairchild Semiconductor), an LED, resistors, and capacitors (see Fig. 3A).

Computer for data acquisition

Data acquisition system (e.g., card PCle-6351, connectors BNC-2090A, and cable SH68-68-EPm from National Instruments)

Dental burr (e.g., round carbide burr FG \#1/4 from Midwest)

Dental drill handpiece (e.g., Tradition from Midwest)

Dura pick (e.g., holder RS-6060 and dissection needle RS-6067 from Roboz Surgical Instrument) Filters

The electrophysiological recording setup requires a high-pass filter (cutoff frequency $300 \mathrm{~Hz}$ ) and a low-pass filter (cutoff frequency $15 \mathrm{kHz}$ ) for a sampling frequency of $40 \mathrm{kHz}$.

Glass capillary (e.g., 3-000-203-G/X from Drummond Scientific)

Heating blanket (for use during anesthesia) (e.g., Homeothermic Blanket System from Harvard Apparatus)

Insect pins (stainless steel) (e.g., 26002-10 from Fine Science Tools)

Isofluorane vaporizer (e.g., Isotec 5 from Datex-Ohmeda)

Light source for stereomicroscope (e.g., KL 1500 LCD from Schott)

Microphone (e.g., AT803 from Audio-Technica) with an amplifier (e.g., MA3 from Tucker-Davis

Technologies) for song recordings

Miniature motorized microdrive (Fee and Leonardo 2001; Otchy and Olveczky 2012) (Fig. 1B)

Motor controller for the microdrive (e.g., MP-285 from Sutter Instrument; modified according to Fee and Leonardo 2001).

Motorized commutator with a torque feedback motor controller (Fee and Leonardo 2001)

Nano connectors (2-pin socket and 2-pin male) (Omnetics Connector; cat. nos. A9069-001 and A9577-001)

Oscilloscope for electrophysiological recording setup (e.g., TDS2024C from Tektronix)

Oscilloscope used during surgical setup (e.g., TBS1062 from Tektronix)

Platinum ground wire (animal ground; 3 mil; PFA-coated) (A-M Systems; cat. no. 772000)

Preamplifier

This apparatus comprises a high-pass filter (cutoff frequency $2.3 \mathrm{~Hz}$ ) followed by an instrumentation amplifier with a gain of 10 (e.g., INA2141U from Texas Instruments) (Fig. 2A, B).

Pulse generator (e.g., Master-8 from AMPI)

Recording electrode mounted on a microdrive for single-unit recording (e.g., 10 megohm quartz/ platinum-iridium fiber electrode from Thomas Recording)

Recording electrode used during surgery to observe spontaneous activity in RA and multiunit "hash" from antidromic stimulation (e.g., Carbostar-1 from Kation Scientific) 
A

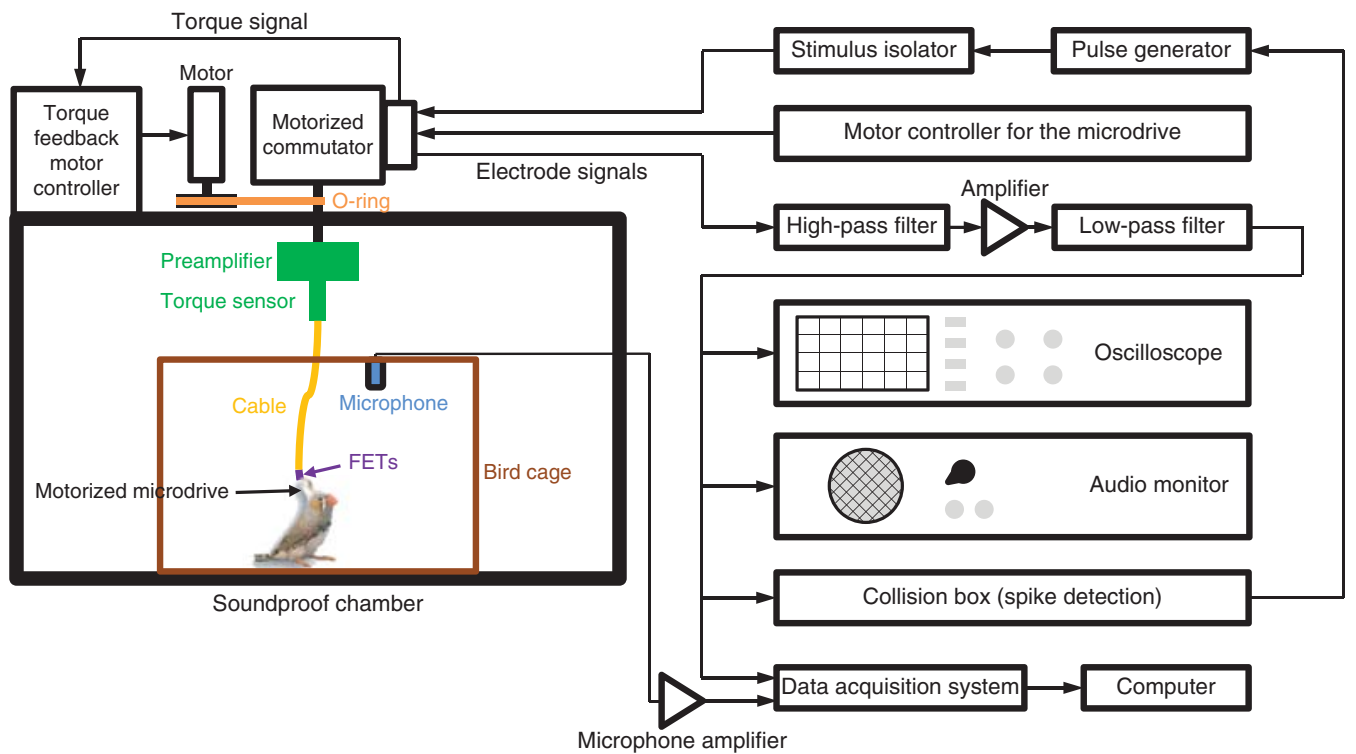

B

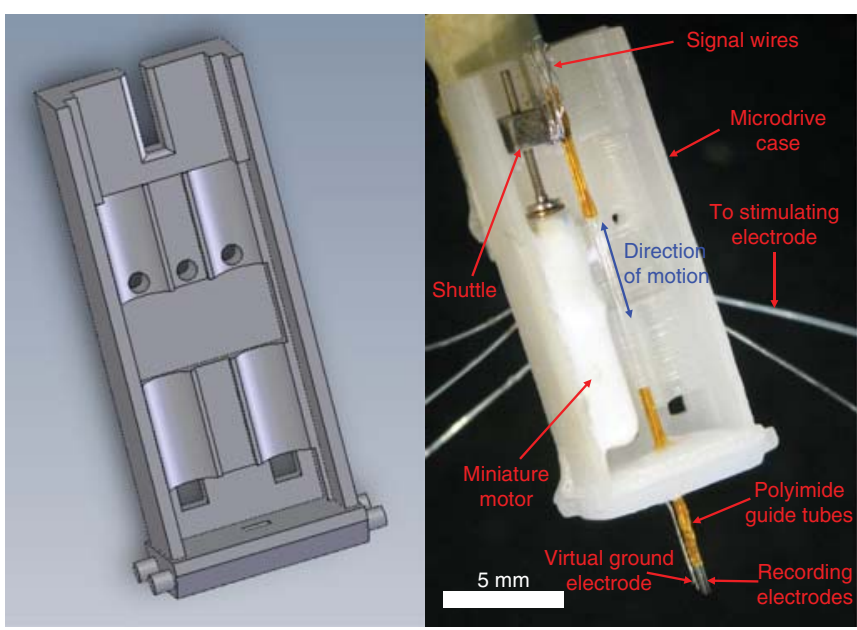

C

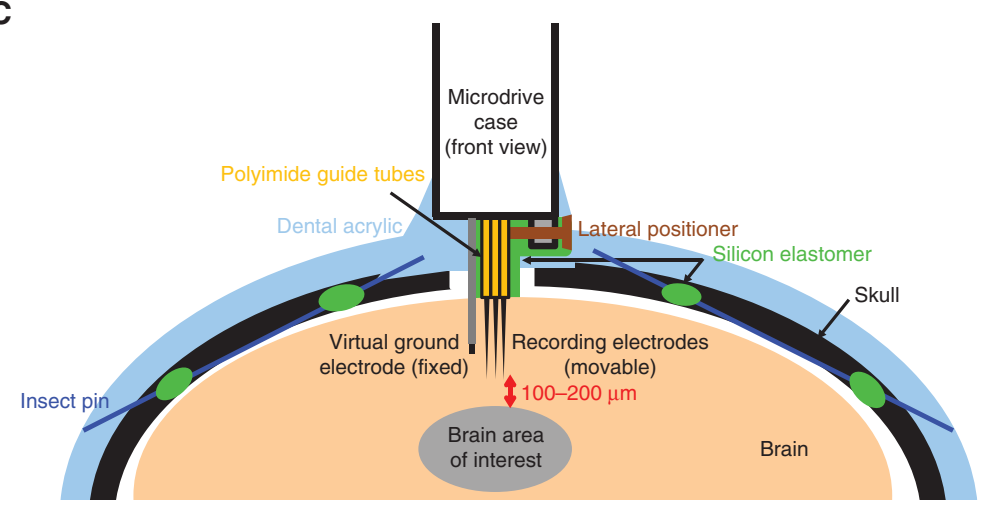

FIGURE 1. Schematic representation of the electrophysiological recording setup and the microdrive implant. (A) The electrophysiological recording setup. For a photograph of the preamplifier and torque sensor, see Figure 2A. For a photograph of the cable, see Figure 2C. (B) Three-dimensional computer-aided design (CAD) drawing (left) and a photograph (right) of a motorized microdrive. The male Omnetics connector is located on the back. The lateral positioner is omitted for clarity but can be seen in $C$. Before the implant, the microdrive is protected by covering the open parts with a transparency film. This CAD drawing is available online as a supplementary material (Microdrive.SLDPRT). (C) Diagram showing the positioning of the microdrive with respect to the skull and the brain. Insect pins go underneath the skull between the two holes in the skull and act as anchors. The lateral positioner provides a way to move the electrodes laterally so that they can enter fresh tissue during each penetration. 
A

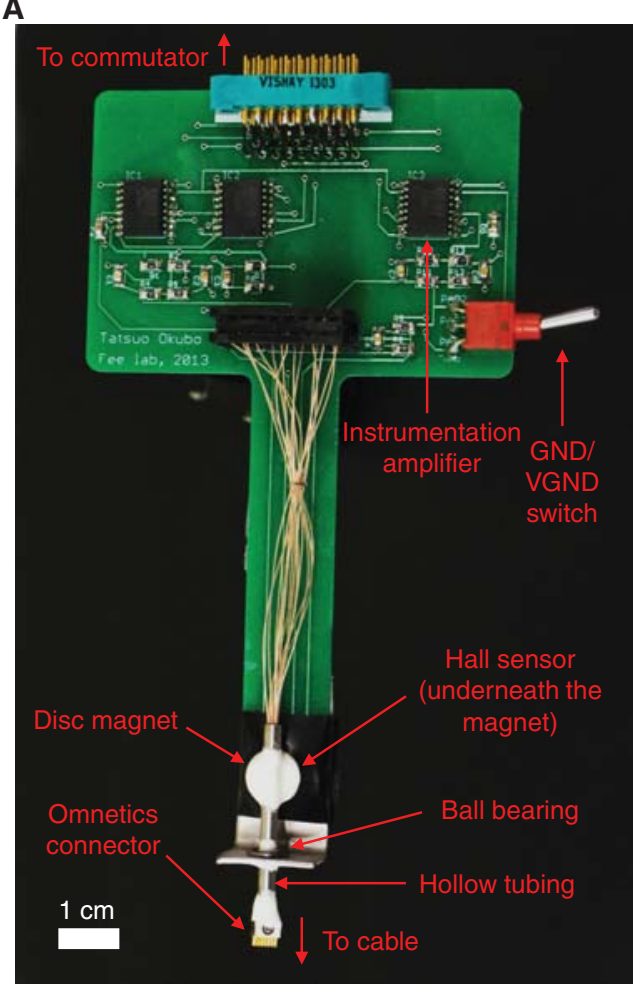

C

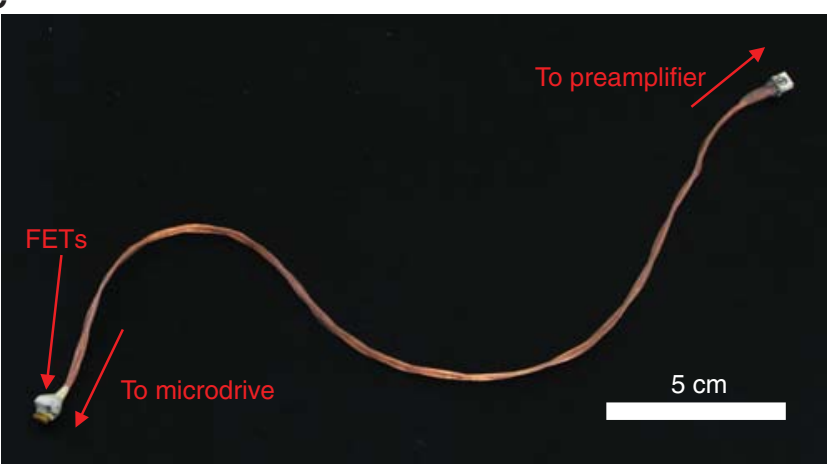

B

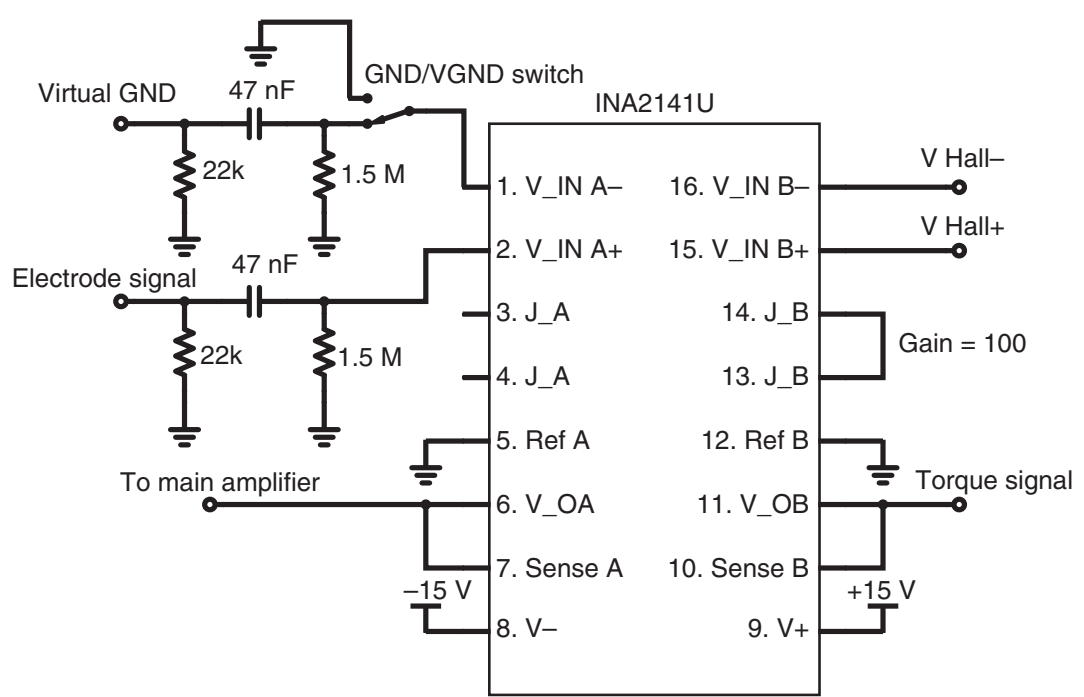

D

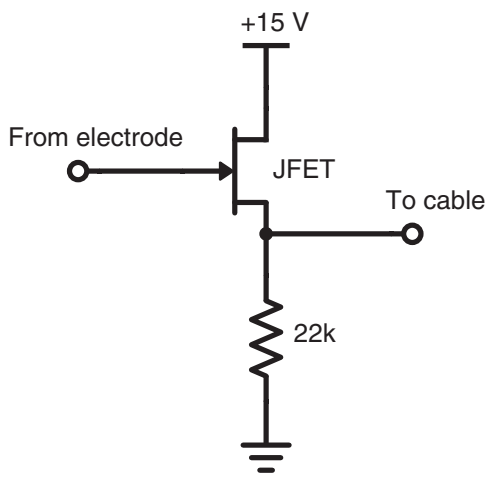

FIGURE 2. Preamplifier and cable. (A) Photograph of the preamplifier and the torque sensor. Rotation of the cable is detected by a Hall sensor placed underneath the disc magnet. The drawing for this printed circuit board is available online as a supplementary material (PreAmp.brd; PreAmp.sch). (B) Diagram of the preamplifier circuit. For simplicity, only one neural channel is drawn. The $47-\mathrm{nF}$ capacitor and 1.5-M $\Omega$ resistor act as a high-pass filter (cutoff frequency $2.3 \mathrm{~Hz}$ ) before the amplification. A $22-\mathrm{k} \Omega$ resistor for the source follower (shown in Fig. 2D) is also included. The GND/VGND switch allows one to select whether GND or VGND is used for the differential amplification. The right half of INA2141U is used for amplifying the torque signal from the Hall sensor. (C) Photograph of the cable. A male Omnetics connector with wires is used at the preamplifier side of the cable. A female Omnetics connector and the surface mount FETs stacked on top of each other are at the microdrive end of the cable. Part numbers are available in the Materials section. The cable is gently braided, and the length of the cable should be such that FETs are $2 \mathrm{~cm}$ above the bottom of the cage when the bird is not plugged in (typically $18-20 \mathrm{~cm}$ ). (D) Schematic of an FET used as a source follower. Each neural channel and the reference ground have their own FET.

Rotary encoder (e.g., encoder E4-300-118, cable CA-FC5-SH-MIC4, and display ED3 from US Digital)

Silver wire (5 mil; PFA-coated) (A-M Systems; cat. no. 786000)

Soldering iron (e.g., soldering station MT1500 and tip MT211 from Weller)

Soundproof chamber (e.g., ENV-018MD-EMS from Med Associates)

Stainless steel microwire (2 mil; PTFE-coated) (California Fine Wire; cat. no. 304) 


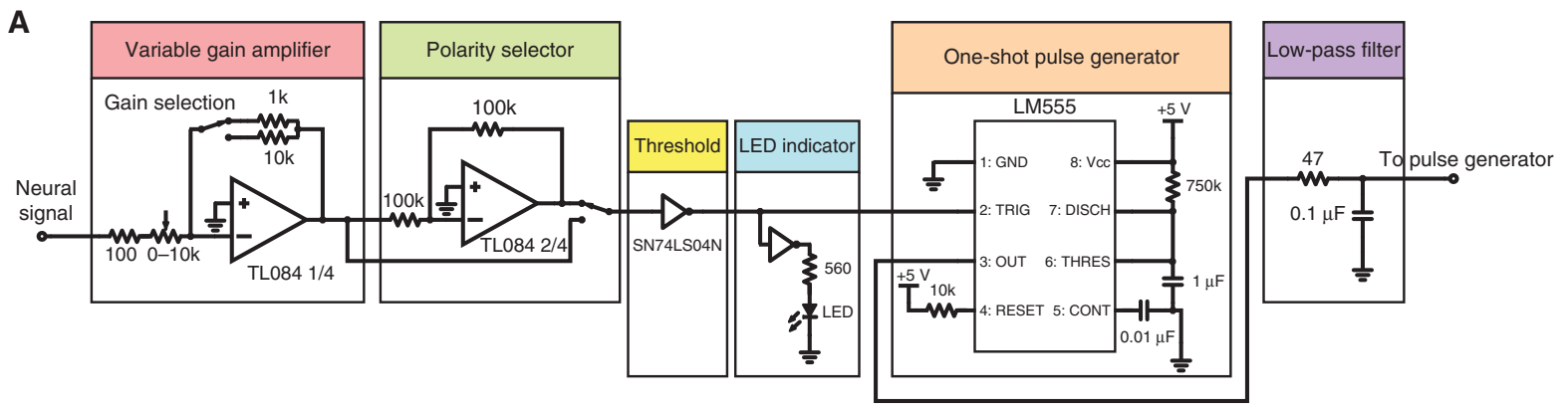

B

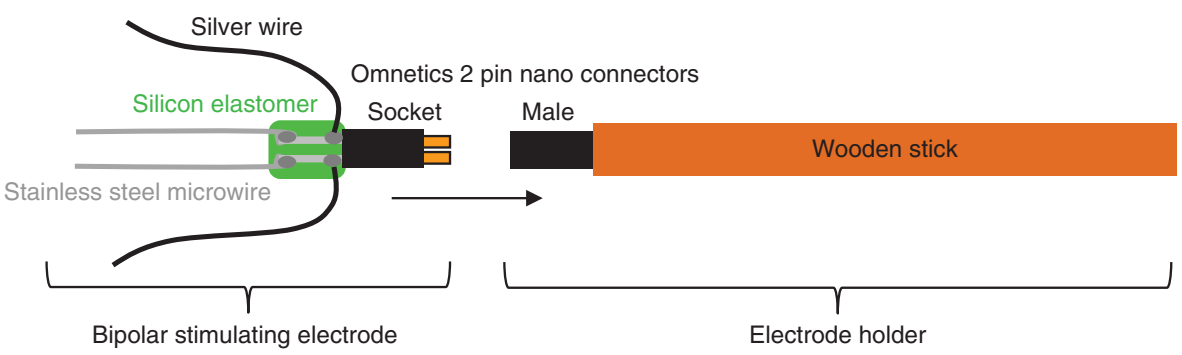

C

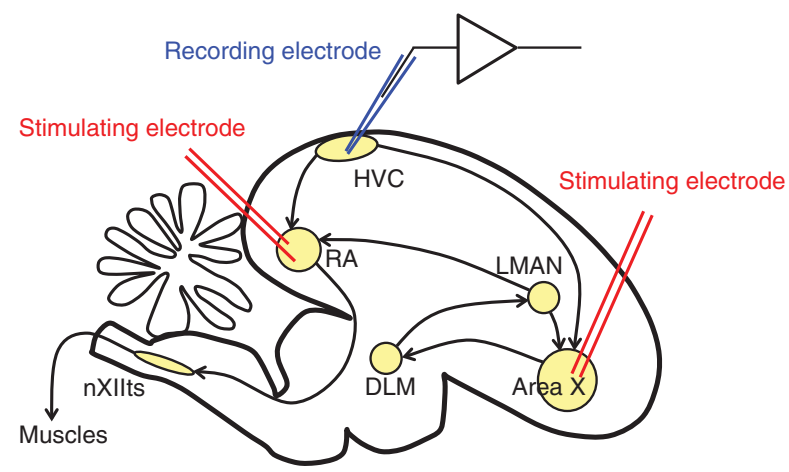

E
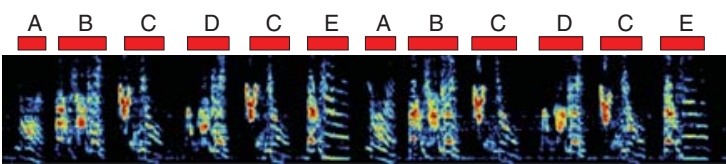

$1 \mathrm{mV}$
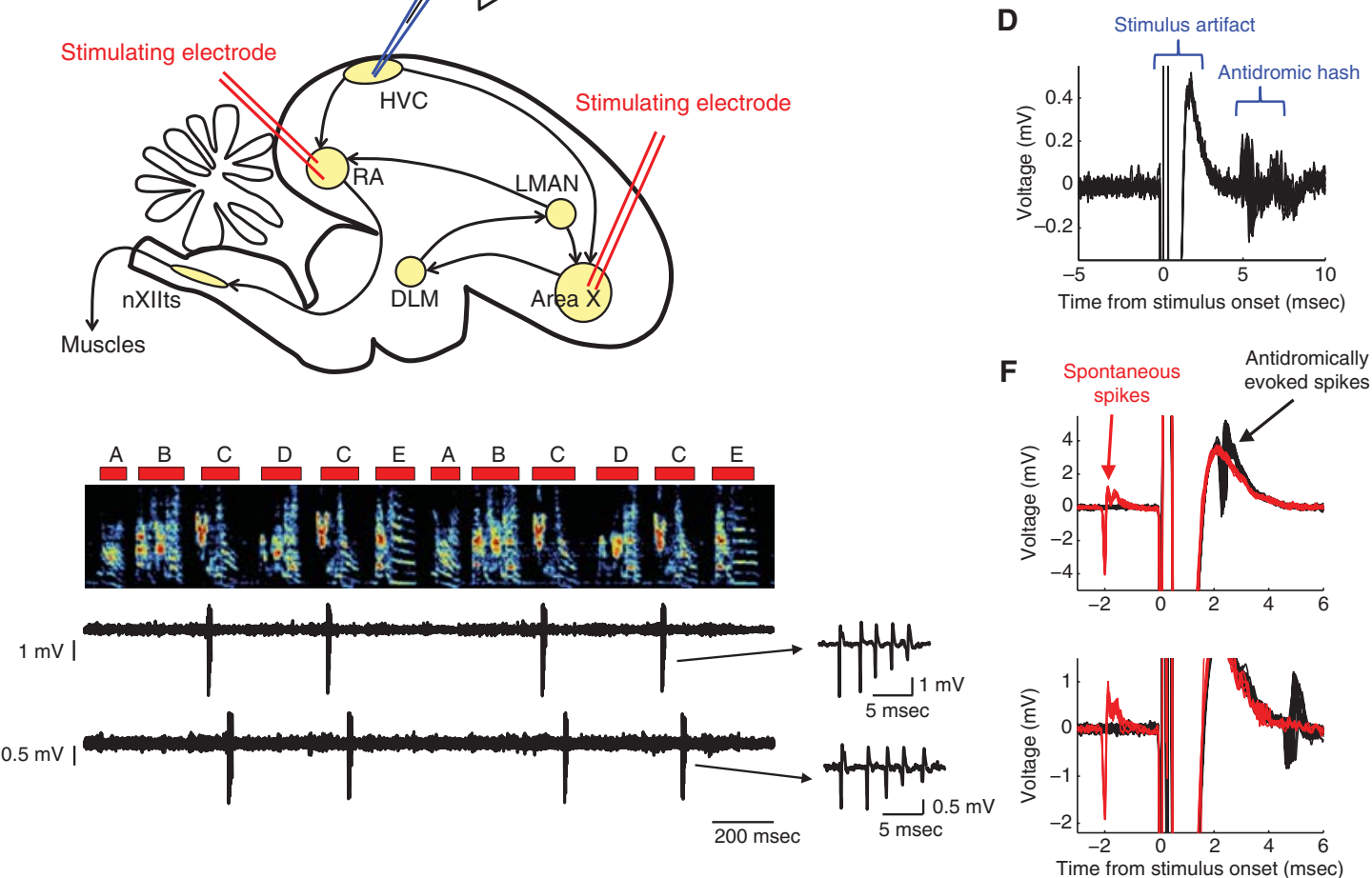

FIGURE 3. Techniques for antidromic identification and example recordings. (A) Diagram for a collision test circuit. The gain and polarity of the amplifier are manually adjusted online depending on the amplitude and polarity of the spike so that they can be detected by crossing the threshold of the inverter. The timer chip LM555 is used in the monostable mode to generate a one-shot pulse to trigger the pulse generator, which triggers the stimulus isolator. It also acts as a hold-off to prevent multiple stimulations faster than $1 \mathrm{~Hz}$. (B) Schematic of the bipolar stimulating electrode and its holder. $(C)$ Schematic of the song system in the zebra finch indicating the locations of bipolar stimulating electrodes in RA and Area X, and a recording electrode in HVC. (D) An example of a multiunit "hash" recorded in HVC following the electrical stimulation in RA (current amplitude $200 \mu \mathrm{A}$ ). Overlay of 20 trials aligned to the onset of the stimulation. $(E)$ An example of a simultaneous single-unit recording of two X-projecting HVC neurons during singing, recorded in an adult male zebra finch. (Top) Spectrogram of the song with syllable labels. (Bottom) Raw voltage traces from two different electrodes. (Right) Enlarged bursts showing individual action potentials. $(F)$ Collision tests for the neurons shown in $(E)$. Voltage traces are aligned to the onset of the electrical stimulation of Area X. Antidromically evoked spikes (black traces) are absent when the stimulation is triggered $2 \mathrm{msec}$ after spontaneous spikes (red traces). 
T.S. Okubo et al.

Stereomicroscope on a boom stand (e.g., MZ9.5 from Leica)

Stereotaxic device (e.g., Digital Stereotaxic Instrument with Fine Drive from Leica Biosystems)

A stereotaxic device designed for mice can be used by replacing the tooth bar with a beak clamp.

Stimulus isolator (e.g., ISO-Flex from AMPI)

Surgical instruments (scalpel, forceps, etc.)

Torque sensor

This apparatus comprises a Hall sensor (e.g., HGT-2101 from Lake Shore Cryotronics), a ball bearing with inner diameter 0.125 in and outside diameter 0.25 in (e.g., BBSRIF-418XXX from Quality Bearings \& Components), a section of hollow stainless steel tubing with outside diameter 0.125 in, an aluminum bracket a disc magnet (e.g. diameter: 0.5 in, thickness: 0.125 in, from Radial Magnets) and a female connector with wires (e.g., A8109-001 from Omnetics Connector) (Fig. 2A, B)

Wire cutter (e.g., 570E from Cooper Hand Tools)

Wooden stick (2 in long) (Fig. 3B)

\section{METHOD}

As an example, we describe procedures for recording from antidromically identified projection neurons in the HVC by placing bipolar stimulating electrodes in the robust nucleus of the arcopallium (RA) and Area X (Hahnloser et al. 2002; Kozhevnikov and Fee 2007), which are the two main projection targets of HVC (Fig. 3C). With minor modifications, the protocol can be utilized to record from other brain areas.

Microdrive Implant Surgery

1. Assemble a bipolar stimulating electrode and its holder. First, construct the electrode holder by attaching a two-pin male nano connector onto a wooden stick (Fig. 3B). Next, construct the electrode by inserting a two-pin socket nano connector into the electrode holder and soldering the stripped ends of a silver wire $(8 \mathrm{~cm}$ in length) and a stainless steel microwire onto each of the two pins of the connector (Fig. 3B). Straighten the stainless steel microwires, make them parallel spaced by $500 \mu \mathrm{m}$, and cut them to an appropriate length ( $3 \mathrm{~mm}$ for RA and $4 \mathrm{~mm}$ for Area X). Cover the pins of the connector and the solder joints using silicon elastomer. Test the stimulating electrode by connecting the silver wires to the stimulus isolator and placing the tip of the electrode in saline solution and passing a small current $(\sim 10 \mu \mathrm{A})$. Bubbles should come out only from the tip of the electrode that is connected to the negative voltage.

The stainless steel microwires will be implanted in the zebra finch brain and the silver wires will connect the stimulating electrode to the microdrive.

The use of special liquid soldering flux is necessary to solder the stainless steel microwires onto the connector pins. It is important to handle the stainless steel microwires with plastic forceps because sharp metal forceps can damage the insulation. This problem can be detected as bubbles coming out from the damaged site during the bubble test.

2. Prepare a microdrive (Fig. 1B) for implant (Fee and Leonardo 2001; Otchy and Olveczky 2012) and make the length of the electrodes so that they will sit at an appropriate depth when implanted (Fig. 1C). Fill the polyimide guide tubes with mineral oil to prevent fluid from entering during the implantation.

The electrodes should be implanted to a depth of a few hundred micrometers above the brain region of interest. At this depth, the polyimide guide tube should be touching the surface of the brain without penetrating it. For HVC, because it is only a few hundred micrometers below the surface of the brain, electrodes can be implanted into HVC and retracted after the implant surgery so that they sit just above HVC until the bird recovers from the surgery.

3. Inject the bird with analgesic $20 \mathrm{~min}$ before the surgery.

4. Place the zebra finch under general anesthesia. For isofluorane, use $3 \%$ for $3 \mathrm{~min}$ for initial induction and maintain at $1 \%-2 \%$.

5. Pluck the feathers on the bird's head.

6. Put the bird on the stereotaxic device. Adjust the ear bars and the beak bar. 
7. Clean the skin on the head with betadine and apply local anesthetic.

8. Make an incision in the skin along the midline with a scalpel and retract the skin. Juvenile skulls are very soft. Avoid damaging the skull during this procedure.

9. Set the head angle to $0^{\circ}$ by placing a glass capillary in the anterior midline groove just behind the beak.

$A O^{\circ}$ head angle corresponds to this glass capillary being horizontal (check with a reference line on the stereotax).

10. Rotate the head forward $20^{\circ}$ using a glass capillary and a protractor or using a rotary-encoded ear bar.

11. Using a dental drill with a round burr, make several pairs of small holes in the skull for the insect pins and one for the ground wire. Drill slowly so as not to damage the dura or the brain underneath.

Insect pins act as anchors for the implant. In addition, bone layers just behind the beak are separated and can be used as an anchor point for dental acrylic. These are not necessary in the adult because the dental acrylic can flow between the two layers of the skull. Make sure the holes are positioned so that the insect pins do not impede access to HVC, RA, or Area X.

12. Carefully thread a pin underneath the skull through each pair of holes. Put a small drop of silicon elastomer to cover each hole (Fig. 1C). Insect pins should go through the holes on the skull tangentially without penetrating the dura.

13. Using one of the holes, tuck the platinum ground wire a few millimeters under the skull so that it is positioned between the skull and the surface of the dura.

The last $1 \mathrm{~mm}$ of the ground wire should be stripped. Make sure that the ground wire is not floating in the air space between the inner and outer leaflets of the skull.

14. Identify the bifurcation of the mid-sagittal sinus (lambda), and use this as a reference point for stereotaxic coordinates.

Juvenile skulls are thin and the mid-sagittal sinus can be seen through the overlying skull. For adults, it is necessary to thin the skull with a dental drill.

15. Implant a stimulating electrode in Area X.

i. Mount the stimulating electrode on the stereotax using the stimulating electrode holder. Make sure the electrode is vertically straight by looking at it from the front and from the side to check the alignment with a reference line on the stereotax. Move the midpoint of the bipolar stimulating electrode to $5.8 \mathrm{~mm}$ anterior, $1.5 \mathrm{~mm}$ lateral.

ii. Make a craniotomy at the coordinates above. Drill through the outer leaflet of the skull. Thin the inner leaflet of the skull with the dental drill and remove it using fine forceps. Remove a small piece of dura using a dura pick.

Juvenile birds have a single-layered skull and thus it is not necessary to drill through the outer leaflet. Once a piece of skull is removed, it is important to prevent the surface of the brain and the dura from drying. The brain can be kept moist by applying saline on the surface every few minutes.

iii. Lower the stimulating electrode to $2.8 \mathrm{~mm}$ deep to target the center of Area X. Advance the electrodes slowly (10 sec/mm).

iv. Cover the exposed part of the craniotomy and the electrode with silicon elastomer. Secure the stimulating electrode by using dental acrylic to connect the insect pins on the skull to the connector on the stimulating electrode. Carefully remove the electrode holder.

16. Implant a stimulating electrode in RA.

i. Rotate the head forward to a head angle of $90^{\circ}$.

ii. Set the recording electrode in the manipulator and make sure it is vertically straight.

iii. Make a craniotomy at $1.8 \mathrm{~mm}$ posterior, $2.4 \mathrm{~mm}$ lateral. Do not remove the dura. 
T.S. Okubo et al.

iv. Using the recording electrode, search for the characteristic spontaneous activity (i.e., regular firing) of RA, which starts $\sim 1.5 \mathrm{~mm}$ beneath the surface. Make several penetrations $200 \mu \mathrm{m}$ apart in the anterior-posterior axis and the medial-lateral axis to map out the extent of RA. Make sure to keep the brain surface moist.

v. Find the center of RA and note its coordinates. Make a scratch on the skull in the vicinity of the craniotomy with a needle and note the coordinates of the center of this mark so that it can be used as a local reference point.

vi. Replace the recording electrode with a stimulating electrode, and by using the local reference, move the stimulating electrodes so that one pole will be in the center of RA.

vii. Remove the dura and implant the stimulating electrode using the same procedure described for Area X.

17. Implant a microdrive in HVC.

i. Make the craniotomy above $\mathrm{HVC}$ at $45^{\circ}$ head angle, $0.4 \mathrm{~mm}$ anterior, and $2.5 \mathrm{~mm}$ lateral. Do not remove the dura.

ii. Hook up the stimulating electrode (either RA or Area X) to the stimulus isolator and start stimulating every second (single pulse, $200 \mu \mathrm{sec}$ duration, $100 \mu \mathrm{A}$ amplitude) using the pulse generator.

iii. Insert the recording electrode in HVC, and search for the antidromic "hash" (Fig. 3D). Make several penetrations $200 \mu \mathrm{m}$ apart, find the center of HVC and note its coordinates. Make a scratch on the skull in the vicinity of the craniotomy with a needle and note the coordinates of the center of this mark.

iv. Replace the recording electrode with a microdrive, and by using the local reference, move the microdrive electrodes to the center of HVC previously identified with the recording electrode.

v. Remove the dura and slowly lower the microdrive electrodes into the brain to the desired depth (Fig. 1C).

Before lowering the electrodes, visually confirm that there is nothing in the way of electrodes and the virtual ground wire, and that the microdrive case will not bump into the stimulus electrodes. At the target depth, the polyimide guide tubes should be touching the surface of the brain (Fig. 1C). Filling the guide tubes with mineral oil before surgery should prevent any blood or fluid from entering the guide tubes, which can dry and prevent the electrodes from moving.

vi. Cover the exposed brain with silicon elastomer.

vii. Secure the microdrive in place by using dental acrylic to connect the microdrive case and the insect pins on the skull (Fig. 1C).

viii. Solder the silver wires from the stimulating electrodes and the ground wire to the appropriate pins on the microdrive connector.

18. Close the incision anterior and posterior to the microdrive by pulling the skin up close to the dental acrylic and applying a small amount of tissue adhesive to the skin using a glass capillary.

19. Carefully remove the bird from the stereotaxic device.

Make sure that the height of the perch in the cage is set so that the microdrive will not get caught.

Single-Unit Recording During Singing

20. Let the bird recover from the surgery for a few days until he starts singing.

21. Place the bird in the recording chamber and plug the cable into the microdrive (Fig. 1A). Turn on the motorized commutator and make sure that it is working properly. Check often that the cable does not become twisted over time. 
22. Slowly lower the electrodes into HVC by moving the motors on the microdrive using the motor controller.

Be careful not to activate the motor for $>1 \mathrm{sec}$ at a time because this can overheat and damage the motor. Advance it with small steps, stopping for a few seconds in between each step.

23. Start stimulating RA or Area $X$ (single pulse, $200 \mu$ sec duration, $100 \mu \mathrm{A}$ amplitude) and observe the antidromic "hash" to confirm that the electrodes are in HVC (Fig. 3D).

See Troubleshooting.

24. When the bird starts singing, isolate a single-unit by slowly moving the electrodes in 10-15 $\mu \mathrm{m}$ steps and waiting for a few minutes in between to check whether there is a single-unit on any of the electrodes. Either search for a single-unit evoked by antidromic stimulation, or isolate a unit during singing and then check the response to antidromic stimulation to confirm its identity.

The current required for the antidromic stimulation is typically within the range of $50-100 \mu \mathrm{A}$, but is occasionally up to $300 \mu \mathrm{A}$.

Interneurons also respond to antidromic stimulation, thus, other criteria (e.g., latency variability of the evoked spike and the spike collision test) will be necessary to distinguish them from projection neurons (Fuller and Schlag 1976; Swadlow 1998; Fee et al. 2004).

See Troubleshooting.

25. If the neuron fires spontaneously, perform a spike collision test by triggering antidromic stimulation off a spontaneous spike with a latency of a few milliseconds. For the projection neurons, the antidromic spike should be blocked (Fig. 3F).

We use a simple electrical circuit to detect threshold-crossing events associated with occurrences of spikes (Fig. 3A). The output of this circuit is used to trigger the pulse generator (e.g., Master-8), which then triggers the stimulus isolator (e.g., ISO-Flex). It is important to have a timer in the circuit (e.g., LM555 timer chip) that prevents multiple stimulations from being triggered, for example, by a burst of spikes. Without this mechanism, the brain will be stimulated for every spike in a burst, which could lead to tissue damage.

26. If the single-unit is lost, advance the electrodes further to find a new unit. Holding time for a single-unit varies from a few minutes to a few hours.

27. Once the electrodes go through the entire depth of HVC, back up the electrodes above HVC.

28. Gently hold the bird, keep the microdrive stable, and turn the lateral positioner on the microdrive so that the electrodes can penetrate into fresh tissue. This is typically done at the end of the day so that the bird has some time to recover from handling.

Lateral positioner is typically rotated $1 / 8$ of a turn, corresponding to roughly $20 \mu \mathrm{m}$.

29. Repeat these procedures over multiple days until the end of the experiment.

Typically, one can perform one or two penetrations per day. To minimize the formation of gliosis in HVC, electrodes are retracted above HVC in between recording sessions.

30. At the end of the experiment, make an electrolytic lesion at the site of recording by passing a negative DC current (15 $\mu \mathrm{A}$ for $10-15 \mathrm{sec})$.

The size of the lesion varies depending on the brain area, and the current parameters need to be adjusted accordingly.

31. Use standard histological techniques to identify the electrolytic lesion and the electrode tracks to verify the recording site.

Problem (Step 23): There is a lack of antidromic responses when the bird is connected to the cable.

Solution: This is likely caused by the misplacement of the microdrive during surgery. It is important to implant the microdrive electrodes at precisely the same location as the site where the antidromic response was found using the recording electrode. To achieve this, we make a scratch on the surface of the skull in the vicinity of the craniotomy as a landmark (Step 17iii). This reduces the 
T.S. Okubo et al.

measurement error by providing a local reference. Alternatively, connect the microdrive to an amplifier during the implant process. This will ensure that the antidromic response is observed before the microdrive is secured to the skull.

Problem (Step 24): During singing, vibration of the skull is picked up by the electrodes and their connecting wires on the microdrive ("microphonics"), which can interfere with neural recordings.

Solution: This issue is detected by listening to the neural channel on the audio monitor because you will hear the song of the bird from this channel. Try securing the electrode connection wires to the microdrive case using a thin layer of acrylic to prevent these wires from picking up vibrations.

Problem (Step 24): Electrical artifacts associated with animal movement are detected.

Solution: Differential recording between the recording electrode and the virtual ground reference electrode (a platinum-iridium wire located in the vicinity of the recording electrode; Fig. 1B, C) helps to remove movement artifacts (Fee and Leonardo 2001). In addition, field-effect transistors (FETs) are used at the microdrive end of the cable (Fig. 2C) in a source-follower configuration for each neural channel and the virtual ground signal (Fig. 2D). The FETs act as unity-gain buffer amplifiers and the signal follows the voltage of the electrodes. This also reduces the signal impedance seen by the cable and makes the signals more robust to noise and artifacts.

\section{DISCUSSION}

The current setup in our laboratory allows an array of five high-impedance electrodes to be positioned by a single motor. Using this configuration, we can obtain single-unit recordings from three to eight projection neurons in a single penetration of HVC, which corresponds to 30-40 recording sites through the $500 \mu \mathrm{m}$ depth of HVC. A simultaneous recording of two neurons is obtained on approximately every other penetration (Fig. 3E, F). One disadvantage of this protocol is that it is difficult to record from a single unit for more than a few hours. An alternative approach using an array of carbon fiber electrodes may improve long-term stability (Guitchounts et al. 2013).

It is possible to combine single-unit recordings during singing with other manipulations. These include fiber tract transection (Olveczky et al. 2011), lesioning (Aronov et al. 2008; Goldberg and Fee 2012; Kojima et al. 2013), and inactivation (Olveczky et al. 2011). Paired recordings from the cell body of a neuron and a calyceal axon terminal (Person and Perkel 2007; Kojima and Doupe 2009; Leblois et al. 2009) have been obtained during singing (Goldberg and Fee 2012). Furthermore, this basic motorized microdrive design has been modified for intracellular recordings during singing (Long et al. 2010).

\section{ACKNOWLEDGMENTS}

We thank Galen F. Lynch for useful discussions and Maya Bronfeld for comments on the manuscript. Funding to M.S.F was provided by the NIH (grant \#R01DC009183 and \#R01MH067105), to T.S.O. by the Nakajima Foundation and the Schoemaker Fellowship, and to E.L.M. by the Department of Defense (DoD) through the National Defense Science \& Engineering Graduate Fellowship (NDSEG) Program.

\section{REFERENCES}

Aronov D, Andalman AS, Fee MS. 2008. A specialized forebrain circuit for vocal babbling in the juvenile songbird. Science 320: 630-634.

Fee MS, Kozhevnikov AA, Hahnloser RH. 2004. Neural mechanisms of vocal sequence generation in the songbird. Ann N Y Acad Sci 1016: $153-170$.
Fee MS, Leonardo A. 2001. Miniature motorized microdrive and commutator system for chronic neural recording in small animals. J Neurosci Methods 112: 83-94.

Fuller JH, Schlag JD. 1976. Determination of antidromic excitation by the collision test: Problems of interpretation. Brain Res 112: 283-298. 
Goldberg JH, Fee MS. 2012. A cortical motor nucleus drives the basal ganglia-recipient thalamus in singing birds. Nat Neurosci 15: $620-627$.

Guitchounts G, Markowitz JE, Liberti WA, Gardner TJ. 2013. A carbonfiber electrode array for long-term neural recording. J Neural Eng 10: 046016.

Hahnloser RH, Kozhevnikov AA, Fee MS. 2002. An ultra-sparse code underlies the generation of neural sequences in a songbird. Nature 419: 65-70.

Kojima S, Doupe AJ. 2009. Activity propagation in an avian basal gangliathalamocortical circuit essential for vocal learning. J Neurosci 29: 4782-4793.

Kojima S, Kao MH, Doupe AJ. 2013. Task-related “cortical” bursting depends critically on basal ganglia input and is linked to vocal plasticity. Proc Natl Acad Sci 110: 4756-4761.

Kozhevnikov AA, Fee MS. 2007. Singing-related activity of identified HVC neurons in the zebra finch. J Neurophysiol 97: 4271-4283.
Leblois A, Bodor AL, Person AL, Perkel DJ. 2009. Millisecond timescale disinhibition mediates fast information transmission through an avian basal ganglia loop. J Neurosci 29: 15420-15433.

Long MA, Jin DZ, Fee MS. 2010. Support for a synaptic chain model of neuronal sequence generation. Nature 468: 394-399.

Olveczky BP, Otchy TM, Goldberg JH, Aronov D, Fee MS. 2011. Changes in the neural control of a complex motor sequence during learning. $J$ Neurophysiol 106: 386-397.

Otchy TM, Olveczky BP. 2012. Design and assembly of an ultra-light motorized microdrive for chronic neural recordings in small animals. J Vis Exp 69: e4314 doi: 10.3791/4314.

Person AL, Perkel DJ. 2007. Pallidal neuron activity increases during sensory relay through thalamus in a songbird circuit essential for learning. $J$ Neurosci 27: 8687-8698.

Swadlow HA. 1998. Neocortical efferent neurons with very slowly conducting axons: Strategies for reliable antidromic identification. J Neurosci Methods 79: 131-141. 


\section{In Vivo Recording of Single-Unit Activity during Singing in Zebra Finches}

Tatsuo S. Okubo, Emily L. Mackevicius and Michale S. Fee

Cold Spring Harb Protoc; doi: 10.1101/pdb.prot084624 originally published online October 23, 2014

\begin{tabular}{rc}
\hline $\begin{array}{r}\text { Email Alerting } \\
\text { Service }\end{array}$ & Receive free email alerts when new articles cite this article - click here. \\
\hline $\begin{array}{c}\text { Subject } \\
\text { Categories }\end{array}$ & $\begin{array}{c}\text { Browse articles on similar topics from Cold Spring Harbor Protocols. } \\
\text { Electrophysiology (104 articles) } \\
\text { Emerging Model Organisms (321 articles) } \\
\text { Neuroscience, general (357 articles) }\end{array}$ \\
\hline
\end{tabular}

